\title{
Modelling architectural visual experience using non-linear dimensionality reduction
}

\author{
Stephan K. Chalup ${ }^{\star}$, Riley Clement, Chris Tucker, and Michael J. Ostwald \\ Faculty of Engineering and Built Environment, The University of Newcastle, \\ Callaghan 2308, Australia
}

\begin{abstract}
This paper addresses the topic of how architectural visual experience can be represented and utilised by a software system. The long-term aim is to equip an artificial agent with the ability to make sensible decisions about aesthetics and proportions when creating its environment. The focus of the investigation is on the feature of line distributions extracted from digital images of house facades. It is shown how non-linear "streetmanifolds" can be calculated where each point on the manifold corresponds to a house façade. Through interpolation between manifold points and the application of an inverse Hough transform basic structure plans for new house façades are obtained. If the interpolated points are close to the manifold it can be argued that the new plans reflect the character of the surrounding streetscape. The method is also demonstrated using basic examples which can be represented by circles.
\end{abstract}

\section{Introduction}

Aesthetical perception is an important factor in understanding the interaction of a living individual with its environment. The discipline of environmental aesthetics argues that the environment is fully integrated with the individual [1] and that "aesthetic values pervade the entire range of human culture" [2] which includes environmental and architectural design of gardens, landscapes, cities, and virtual space.

The concept of streetmanifolds was introduced in $[5,6]$ to provide a holistic geometrical representation of the visual experience which can be gained through evaluation of a large set of house façades. Navigation in the streetmanifold would correspond to continuous morphing and interpolating between façade designs represented by the data set of images of house façades. The concepts of holism, continuity, and clustering are associated with manifold learning [26, 29] but can also be found in Gestalt psychology $[18,30]$ which has close links to the concepts of visual neuroscience [7].

The hypothesis of the present study is that the visual experience gained by an architect through visual perception of thousands of house façades during his education and professional life may be captured in a structure which corresponds to some form of streetmanifold.

\footnotetext{
* Email: Stephan.Chalup@newcastle.edu.au, phone: +61 249216080
} 


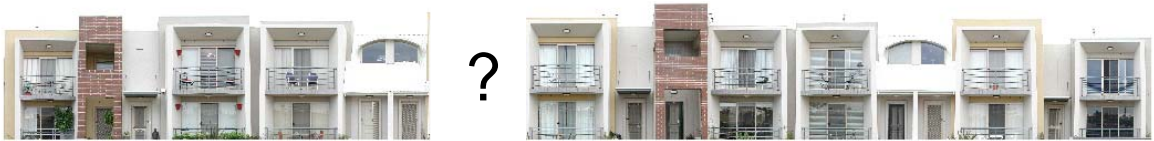

The house façades along a street contribute to the character of a streetscape [9, $27,28]$. This is an important factor for architects who design a new house for an empty spot between the other houses of a street such that the new house harmonically relates to the neighbourhood [12]. The present study's streetmanifolds are based on the calculation of pairwise distances between digital images of house façades. At the current stage of the project the focus is on an important feature in the visual perception of houses which is the distribution of lines determined by the edges of the main components of a house façade. Typically most of the lines have horizontal or vertical direction with a few approximately diagonally oriented lines along the roof or gable. Figure 6 shows examples of house façades with virtual lines along edges extracted using a Hough transform [14, 24].

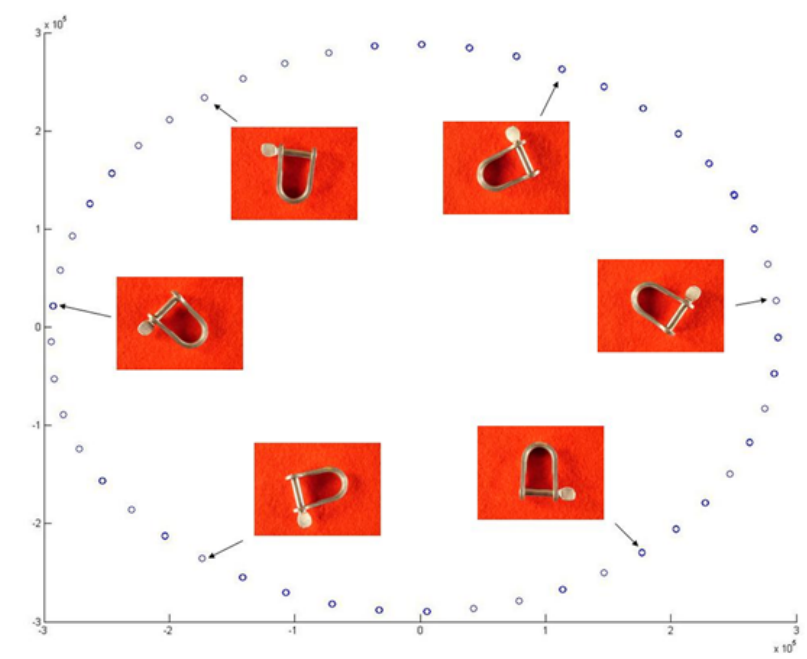

Fig. 1. Circle extracted from images of a rotating shackle using 4-isomap.

The approach to take line directions as the central feature for the calculation of streetmanifolds is supported by research in visual neuroscience which found that detection of edge directions is a key component of the human visual system $[15,19]$. It also was found that the visual system has specialised areas for representation of different entities such as buildings [11].

The main new contribution of the present article is to utilise the streetmanifolds calculated from our dataset of house façades in [5] to generate basic plans for new house façades. The new plans are distributions of lines which are obtained 
through linear interpolation of points on the streetmanifold and application of an inverse Hough transform.

Previous related work which addresses how artificial life methods can be applied in architecture include philosophical discussions [21] or software development associated with the area of emergent design [13, 20,22]. Reich [21] addressed the topic of how aesthetic judgment can be incorporated in computational design. He claimed that aesthetic criteria are embedded in designers' expertise and their use is manifested in existing designs. Reich discussed how rationalistic and romanticistic aesthetic criteria can synergistically be applied to design. As practical example a system for the design of cable-stayed brides was presented. Frazer [10] proposed a generative design tool for architects based on cellular automata. An artificial life based emergent design software system was developed by Ross et al. [22]. It allows architects to endow elements of an architectural scenario with agency and dynamic spatial interaction. Hemberg et al. [13] developed computational generative design software for architects which can generate three dimensional forms and surfaces. Their system used evolutionary algorithms and L-systems grammars. The aim was to be able to grow and evolve organic forms.

The remaining sections of this paper address the topic of manifold learning (section 2), some basic examples of learning circle manifolds (section 3), the procedure how to extract a streetmanifold from a set of digital images of house façades (section 4), and how to generate plans for new houses through interpolation of manifold points (section 5). In section 6 follows a brief discussion and summary of the results.

\section{Manifold learning}

Manifolds are locally Euclidean spaces with some additional very general mathematical properties [25]. In dimension one they appear as continuous deformations of lines and circles and in dimension two they are surfaces derived from spheres, tori, pretzel surfaces, or similar objects. The manifold concept generalises to higher dimensions.

Manifold learning describes algorithms for non-linear dimensionality reduction $[4,23]$. The aim of manifold learning algorithms is to detect the essential underlying geometric structure of a high-dimensional data set, to extract it as a low-dimensional manifold and to embed it faithfully into a low-dimensional space.

In contrast to the relatively new manifold learning techniques traditional methods for dimensionality reduction such as principal component analysis (PCA) [16] or multidimensional scaling (MDS) [8] were designed for reducing the dimensionality of data when the underlying structure was linear.

Two manifold learning methods, isomap [26] and maximum variance unfolding (MVU) [29], have been employed in the present project to calculate streetmanifolds [5]. Both methods can be applied by first calculating a distance matrix based on a weighted $k$-nearest neighbour graph of the data points. 


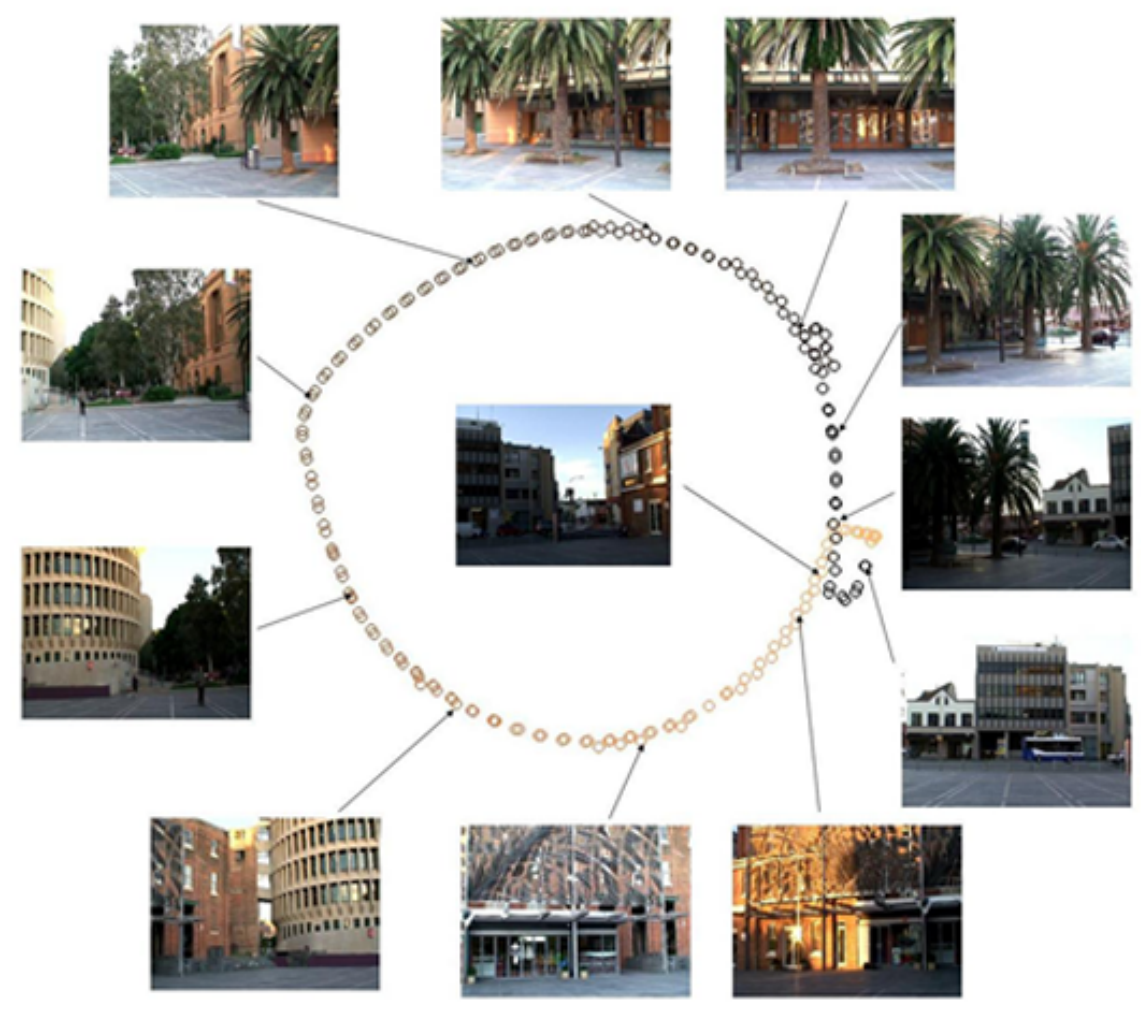

Fig. 2. Circle extracted by 4 -isomap from images taken by an HD video camera rotating about $360^{\circ}$ in the middle of Wheeler place in Newcastle. For the experiment about 200 overlapping frames were extracted from the video sequence. Twelve of them are displayed above together with the corresponding points on the circle manifold.

In isomap [26] these pairwise distances, which can be regarded as approximations to geodesic distances on the manifold, are fed into a MDS. That is, isomap can be regarded as a modification of MDS where instead of the euclidean distances approximation to geodesic distances are used. MDS then tries to map the data into a lower dimensional space while preserving the pairwise distances [8].

The aim of MVU $[29,23]$ is to maximise the sum of pairwise distances of all data points, i.e. $\sum_{i j}\left(\left\|y_{i}-y_{j}\right\|^{2} \cdot \delta_{N N}\left(x_{i}, x_{j}\right)\right)$, where $\delta_{N N}\left(x_{i}, x_{j}\right)$ is 1 if $x_{i}$ and $x_{j}$ are nearest neighbours and 0 otherwise; The maximisation is subject to two conditions which postulate that: (I) distances between nearest neighbour inputs should be the same as between the associated outputs, i.e. $\left\|y_{i}-y_{j}\right\|^{2}=\left\|x_{i}-x_{j}\right\|^{2}$ and (II) the outputs should be centered at the origin, i.e. $\sum_{i} y_{i}=0$. 


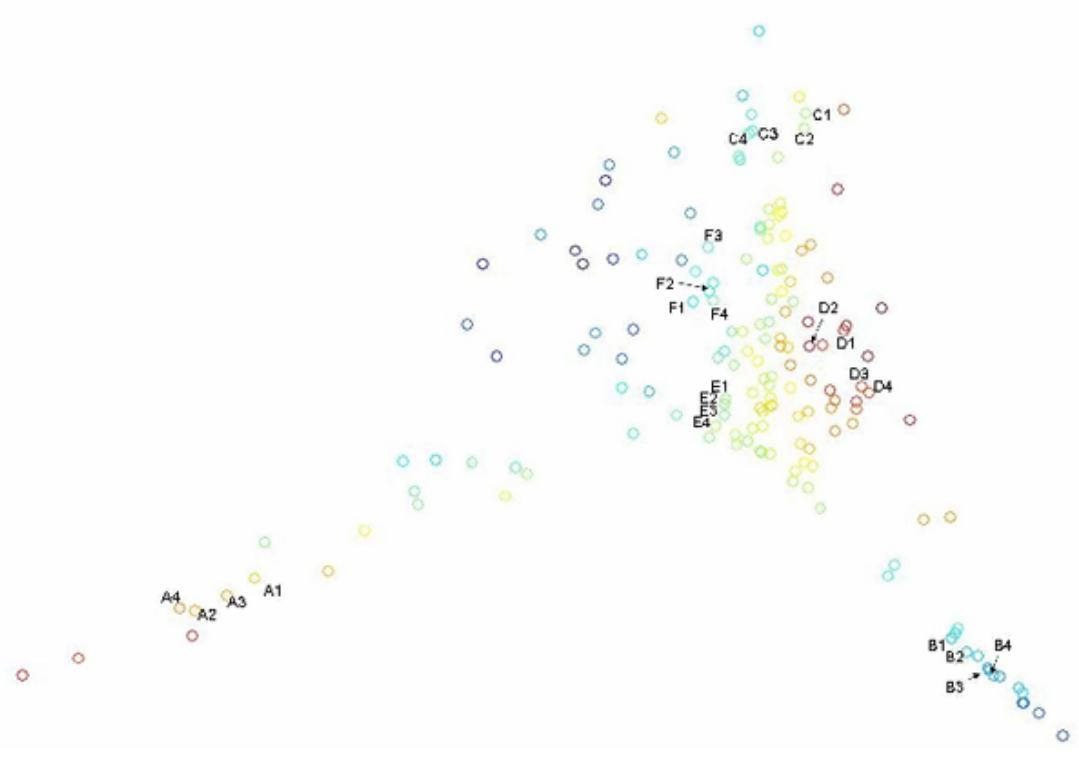

Fig. 3. Streetmanifold calculated with isomap and Bhattacharyya based distance. Colour encodes the third dimension.

\section{$3 \quad$ Extracting circle manifolds}

A simple example how manifold learning works is shown in figure 1. Given was a sequence of digital images of a rotating shackle, i.e. the underlying dynamics of the data set was a rotation. The dimension of the space of digital images is the number of pixels in each image, i.e. $192 \times 292$. Isomap with $k=4$ was able to extract a 1-dimensional circle, that is, a non-linear 1-dimensional manifold embedded in $\mathbf{R}^{\mathbf{2}}$ from the rotating shackle data.

In a second experiment, instead of taking pictures of a rotating object, we rotated the camera at the center point of a circle. Figure 2 shows that the result 4 -isomap extracted from an image sequence taken by an HD video camera while rotated in the middle of Wheeler place in Newcastle is again a circle. The data consisted of about 200 overlapping frames sampled from the video sequence.

\section{Calculating streetmanifolds}

The calculation of the streetmanifolds was based on a dataset of several hundred digital images of house façades which were taken in Newcastle and selected by a team of researchers from architecture. Some example images are shown in figure 6 . 


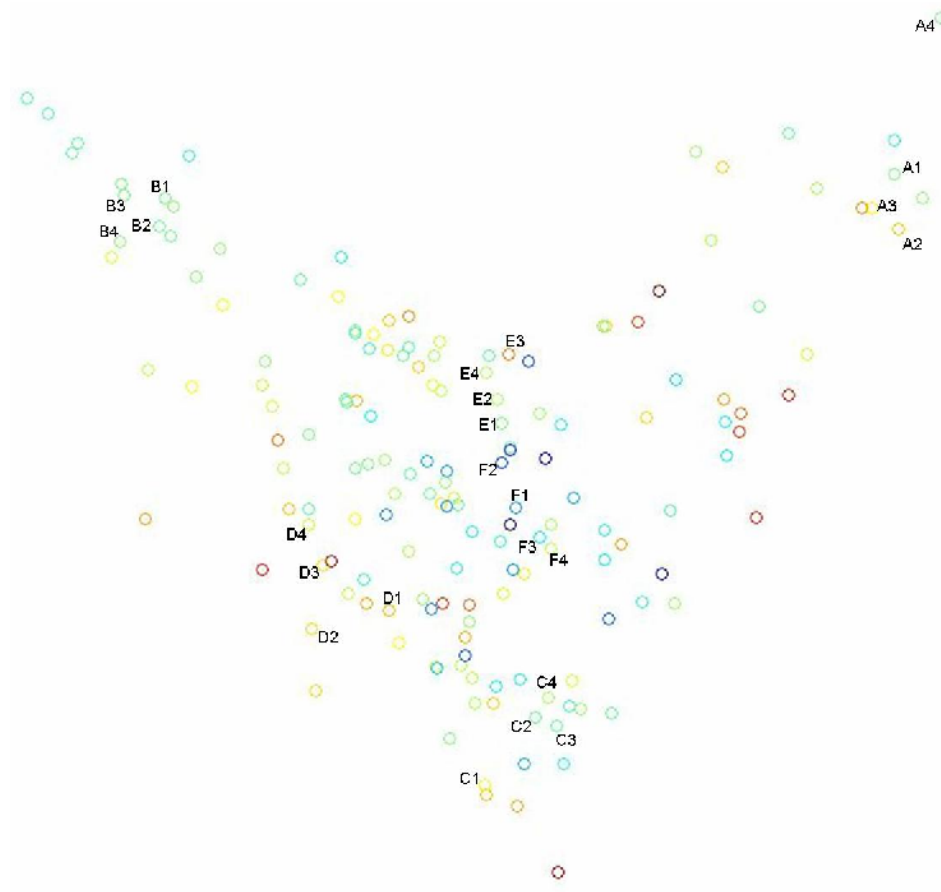

Fig. 4. Streetmanifold calculated with MVU and Bhattacharyya based distance. The manifold is very similar to figure 3 .

A line can be regarded as a set of points $\mathbf{x}=\left(x_{1}, x_{2}\right) \in \mathbf{R}^{2}$ and can be determined by using the Hessian normal form $\left\{\mathbf{x} \in \mathbf{R}^{2} ;[\cos \varphi, \sin \varphi] \cdot \mathbf{x}-b=0\right\}$, where $\varphi \in\left[0,360^{\circ}\right.$ [ controls the slope of the line's normal vector and $b \in \mathbf{R}$ is its perpendicular distance from the origin. Using the Hough transform [14,24] each image was associated with an array of discrete parameters $(\varphi, b) \in\left[0,360^{\circ}[\times \mathbf{R}\right.$ the Hough array - where each point corresponds to a line in the image.

For the application of isomap and MVU the distance between each pair of Hough arrays was calculated. The discrete set of point values in the Hough arrays was smoothed by multiplying each point in the array with a Gaussian function. Then for each pair of smoothed Hough arrays $A=\left(a_{i j}\right)_{\substack{i=1, \ldots, m \\ j=1, \ldots, n}}$ and $B=\left(b_{i j}\right)_{\substack{i=1, \ldots, m \\ j=1, \ldots, n}}$ their Euclidean distance was calculated using $d_{2}(A, B)=$ $\left(\sum_{\substack{i=1, \ldots, m \\ j=1, \ldots, n}}\left(a_{i j}-b_{i j}\right)^{2}\right)^{1 / 2}$. An alternative distance based on the Bhattacharyya distance measure $[3,17]$ was applied after normalisation of the arrays: $d_{B h a t}(A, B)=$ $1-\sum_{\substack{i=1, \ldots, m \\ j=1, \ldots, n}} \sqrt{a_{i j}} \sqrt{b_{i j}}$.

Application of isomap or MVU allowed to embed the manifold of Hough arrays into two or three-dimensional space (figures 3, 4, and 5). 


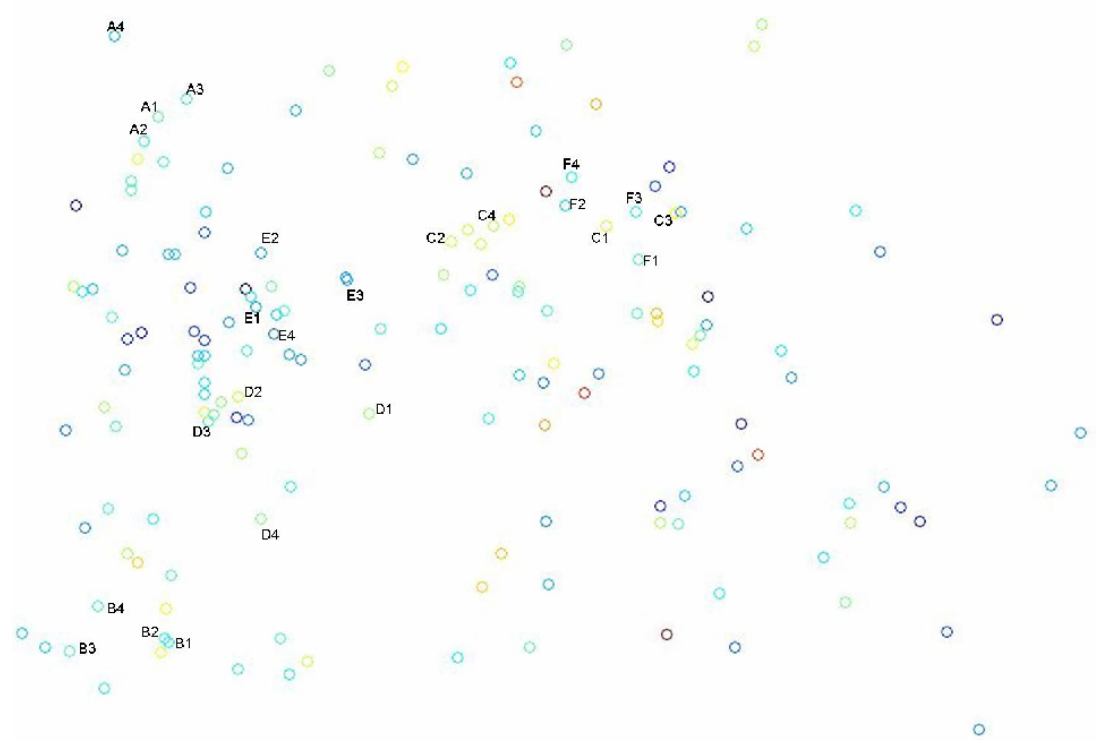

Fig. 5. Streetmanifold calculated with MVU and Euclidean distance appears to have a different shape but shows similar clusters as figures 4 and 3. Colour encodes the third dimension.

To evaluate the streetmanifold we selected six clusters of houses (A-F) in figures 3,4 , and 5 . Four representative houses from each of the six clusters are shown in figure 6 . We found (cf. [5]) that houses of category A were narrow and had a relatively high percentage of vertical lines. In contrast the houses of category B were wide and had strong horizontal and vertical components. Category $\mathrm{C}$ had houses of medium width with many horizontal lines. The D category was very similar to the $\mathrm{C}$ category but the houses were wider in $\mathrm{D}$. In the $\mathrm{E}$ category houses were hidden behind trees and the distribution of horizontal and vertical lines tended to be homogeneous. The associated cluster was located at a close to central position. Cluster F contained houses with average characteristics.

\section{Generating design templates for new house façades through linear interpolation of streetmanifold points}

The geometry of the streetmanifold is determined by the distances between all records of the dataset. Therefore the streetmanifold calculated from the image dataset of the houses of a street or neighbourhood can be regarded as a representation of the aesthetical character of the streetscape.

Points on or close to the streetmanifold represent Hough arrays of façades which have similar features as those of the images which were used to generate 


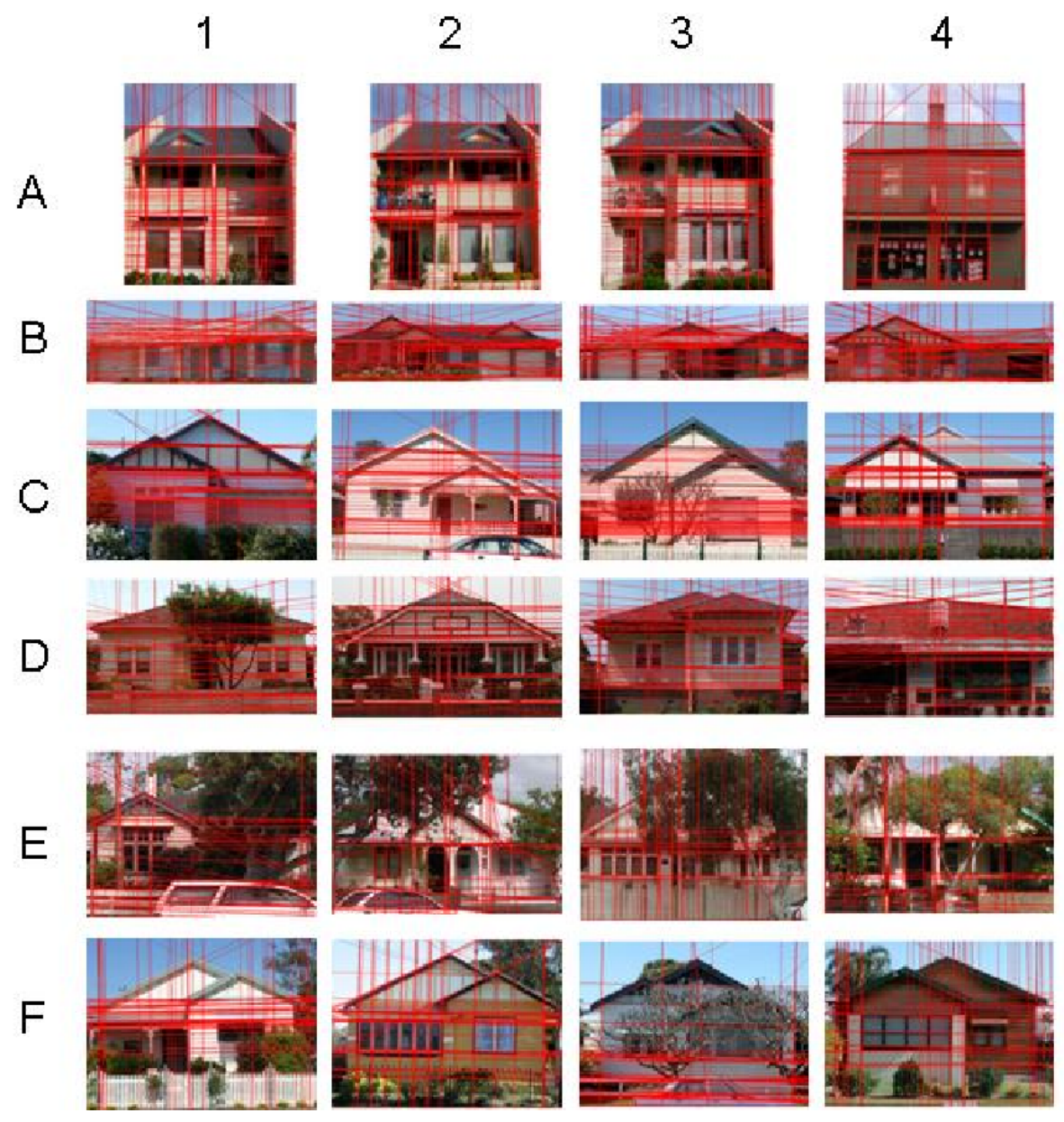

Fig. 6. Six clusters of houses (A-F) found in the streetmanifolds.

the manifold. Through application of an inverse Hough transform it is possible to generate for each manifold point a line distribution as shown in the middle column of figure 7 . These patterns of lines may be used as a plan for architects to outline basic proportions of a house which should fit into the streetscape.

In the present study several pairs of house facades were selected and for each pair a linear interpolation of the associated Hough arrays was calculated. Then an inverse Hough transform was applied to the result of the interpolation. Before interpolation the Hough arrays were smoothed by multiplying each peak with a Gaussian function. The inverse Hough transform was calculated by selecting the 30 highest local maxima of the sum of the two smoothed Hough Arrays. 

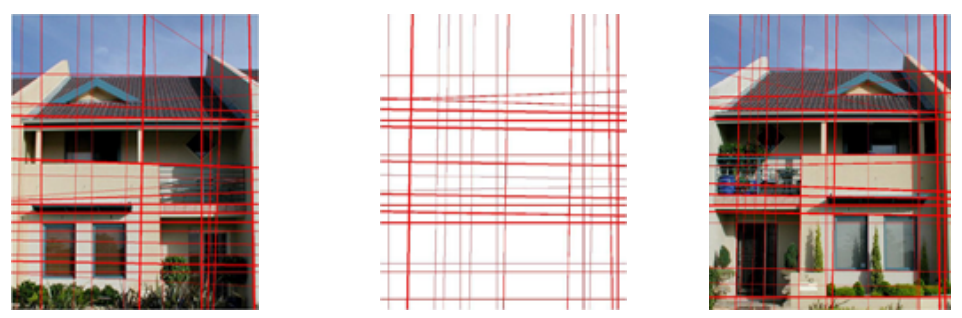

Interpolation of A1 and A2
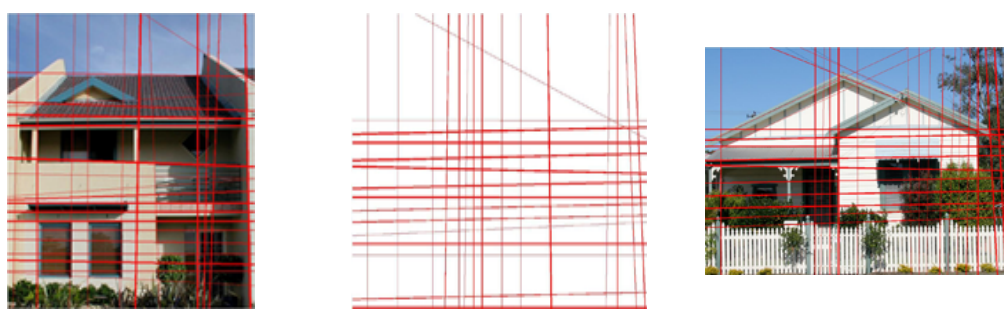

Interpolation of A1 and F1
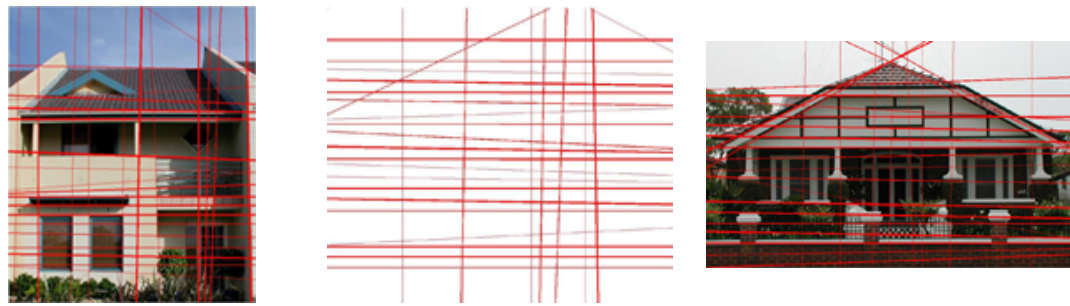

Interpolation of A1 and D2
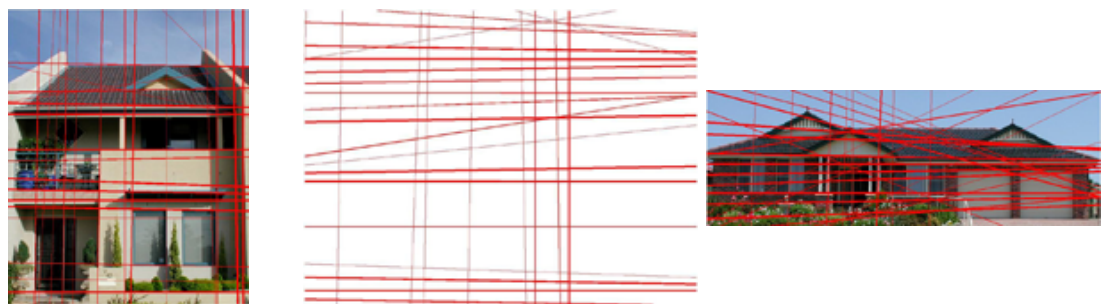

Interpolation of A2 and B2
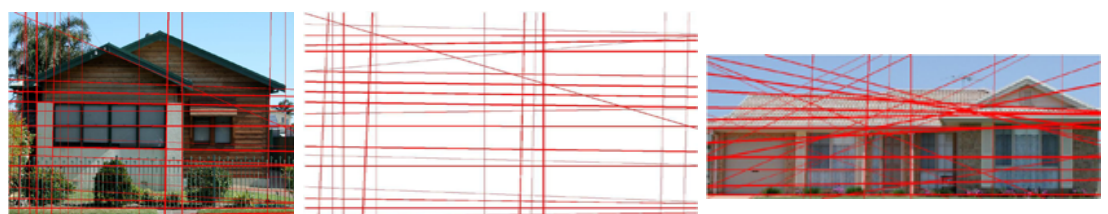

Interpolation of F4 and B1

Fig. 7. The middle column shows the inverse Hough transforms of interpolations between Hough arrays corresponding to five pairs of house façades. The outcome indicates that interpolation between A1 and A2 or A1 and F1 led to sensible results in contrast to interpolation between distant points such as A2 and B2. 
Figure 7 shows in the middle column the resulting plans obtained by this procedure of interpolation between Hough arrays of the pairs (A2, B2), (F4, B1), (A1, A2), (A1, D2), and (A1, F1), respectively. The house façades were selected from the data used to calculate the streetmanifolds and are also displayed in figure 6 . The format and size of the plans in the middle column of figure 7 was determined by taking the maximum of the two heights and widths of the two images of the house façades which were used in the interpolation process.

\section{Discussion and summary}

The streetmanifolds in figures 4 and 5 show a comparable structure of clusters to the streetmanifolds of figure 3 and our previous results [5]. The resulting clusters suggest that the streetmanifolds have captured and smoothly organised a variety of line-based features of the whole data set in one object.

Although streetmanifolds are non-linear we have employed linear interpolations of smoothed Hough arrays. That means that for close points such as A1 and A2 the interpolation result is likely to be close to the manifold but for distant points such as A2 and B2 the interpolation result may be far outside the manifold and hence not representative for the character of the streetscape.

In some cases we added the interpolation result to the initial data set of Hough arrays an recalculated the streetmanifolds. For close points the manifold did not change much but for some of the distant points the newly calculated streetmanifolds seemed to have changed significantly compared to the original.

These results seem to support the hypothesis that local interpolation (e.g. between A1 and A2) or interpolation on the manifold may lead to plans which are conform with the character of the streetscape which is represented by the geometry of the streetmanifold.

Future research may investigate alternative options of interpolation on streetmanifolds and their use in software systems for generative design.

\section{References}

1. Berleant, A.: The Aesthetics of Environment. Temple University Press (1995)

2. Berleant, A.: Aesthetics and Environment: Theme and Variations on Art and Culture. Ashgate Publishing, Limited (2005)

3. Bhattacharyya, A.: On a measure of divergence between two statistical populations defined by their probability distributions. Bulletin of the Calcutta Mathematical Society 35 (1943) 99-109

4. Burges, C.J.C.: Geometric Methods for Feature Extraction and Dimensional Reduction. In: Data Mining and Knowledge Discovery Handbook: A Complete Guide for Researchers and Practitioners. Kluwer Academic Publishers (2005)

5. Chalup, S.K., Clement, R., Marshall, J., Tucker, C., Ostwald, M.J.: Representations of streetscape perceptions through manifold learning in the space of hough arrays. In: 2007 IEEE Symposium on Artificial Life, April 1-5, 2007. (2007) 
6. Chalup, S.K., Clement, R., Ostwald, M.J., Tucker, C.: Applications of manifold learning in architectural façade and streetscape analysis. Workshop on Novel Applications of Dimensionality Reduction at NIPS, Whistler CN 2006 (2006) extended abstract.

7. Chalupa, L.M., Werner, J.S., eds.: The Visual Neurosciences. The MIT Press (2004)

8. Cox, T.F., Cox, M.A.A.: Multidimensional Scaling. second edn. Chapman \& Hall/CRC (2001)

9. DIPNR: Neighbourhood Character. Sydney: NSW Department of Infrastructure Planning \& Natural Resources. (2004)

10. Frazer, J.: An Evolutionary Architecture. Architectural Association, London (1995)

11. Grill-Spector, K., Malach, R.: The human visual cortex. Annual Reviews Neuroscience 27 (2004) 649-677

12. Groat, L.: Contextual compatibility in architecture: An issue of personal taste? In Nasar, J., ed.: Environmental aesthetics: Theory, research, and applications. Cambridge University Press (1988) 228-253

13. Hemberg, M., O'Reilly, U.M., Menges, A., Jonas, K., Goncalves, M., Fuchs, S.: Exploring generative growth and evolutionary computation for architectural design. In Machado, P., Morelo, J.J., eds.: Art of Artificial Evolution. Springer (2006)

14. Hough, P.V.C.: Methods and means for recognizing complex patterns. U.S. Patent 3,069,654 (1962)

15. Hubel, D.H., Wiesel, T.N.: Receptive fields, binocular interaction, and functional architecture in the cat's visual cortex. Journal of Physiology (London) 160 (1962) 106-154

16. Jolliffe, I.T.: Principal Component Analysis. Springer-Verlag, New York (1986)

17. Kailath, T.: The divergence and bhattacharyya distance measures in signal selection. IEEE Transactions on Communication Technology 15 (1967) 52-60

18. Koffka, K.: Principles of Gestalt Psychology. New York: Harcourt Brace (1935)

19. Larsson, J., Landy, M.S., Heeger, D.J.: Orientation-selective adaptation to firstand second-order patterns in human visual cortex. Journal of Neurophysiology 95 (2005) 862-881

20. O'Reilly, U.M., Hemberg, M., Menges, A.: Evolutionary computation and artificial life in architecture: Exploring the potential of generative and genetic algorithms as operative design tools. Architectural Design 74 (2004) 48-53 Special Issue on Emergence.

21. Reich, Y.: A model of aesthetic judgment in design. Artificial Intelligence in Engineering 8 (1993) 141-153

22. Ross, I., O'Reilly, U.M., Testa, P.: Emergent design: Artificial life for architecture design. (2000)

23. Saul, L.K., Weinberger, K.Q., Sha, F., Ham, J., Lee, D.D.: Spectral methods for dimensionality reduction. In Chapelle, O., Schölkopf, B., Zien, A., eds.: SemiSupervised Learning. The MIT Press, Cambridge, MA (2006) 293-308

24. Shapiro, L.G., Stockman, G.C.: Computer Vision. Prentice Hall (2001)

25. Spivac, M.: A Comprehensive Introduction to Differential Geometry. second edn. Publish or Perish, Inc. (1979)

26. Tenenbaum, J.B., de Silva, V., Langford, J.C.: A global geometric framework for nonlinear dimensionality reduction. Science 290 (2000) 2319-2323

27. Tucker, C., Ostwald, M.J., Chalup, S.K.: A method for the visual analysis of streetscape character using digital image processing. In Bromberek, Z., ed.: Contexts of 
Architecture: Proceedings of the 38th Annual Conference of the Architectural Science Association ANZAScA and the International Building Performance Simulation Association, Launceston, Tasmania: Australia and New Zealand Architectural Science Association (2004) 134-140

28. Tucker, C., Ostwald, M.J., Chalup, S.K., Marshall, J.: Sustaining residential social space: a visual and spatial analysis of the nearly urban. In: ANZAScA 40th Annual Conference of the Architectural Science Association, "Challenges for architectural science in changing climates", 22-25 November 2006, The University of Adelaide Adelaide, South Australia. (2006)

29. Weinberger, K.Q., Saul, L.K.: An introduction to nonlinear dimensionality reduction by maximum variance unfolding. In: Proceedings of the National Conference on Artificial Intelligence (AAAI), Nectar paper, Boston MA (2006)

30. Wertheimer, M.: Untersuchungen zur Lehre von der Gestalt II. Psychologische Forschung 4 (1923) 301-350 